\title{
PREVALENCE OF SCHNEIDERIAN MEMBRANE PERFORATION DURING MAXILLARY SINUS AUGMENTATION PROCEDURES USING ULTRASOUND VERSUS ROTARY TECHNIQUES
}

\author{
Gamal M. Moutamed*
}

\begin{abstract}
Schneiderian membrane perforation is one of the main complications during sinus augmentation. The aim of the study was to investigate sinus membrane rupture in maxillary sinus lifting surgery with the ultrasound and with conventional rotary technique, analyzing the bone gain obtained after the operation using cone beam computed tomography (CBCT), and examining the postaugmentation survival of implants. Twenty-two patients requiring dental implant therapy in the posterior maxillary region with insufficient bone volume were included in the study. Patients were randomly and equally allocated to Piezosurgery group and the conventional rotary group. A total of 22 sinus lifting surgeries were carried out; 11 with ultrasound in piezosurgery group and 11 with DASK drills in conventional rotary group. A total of 47 dental implants were placed three months after sinus augmentation. Patients were randomly and equally allocated to Piezosurgery group and the conventional rotary group. In either Piezosurgery group or conventional group a total of 11 sinus lifting surgeries in 11 patients were performed. The bone gain obtained as a result of sinus lift was calculated using CBCT scanning. Perforations of Schneider's membrane with the ultrasound and rotary technique occurred in $9 \%$ and $18 \%$ of the cases, respectively, with membrane integrity being preserved in $86.3 \%$ of operated patients. All 47 implants placed showed the implant success criteria throughout postoperative follow-up in the both study groups. No statically significant difference in bone gain was recorded between study groups. In conclusions, perforations of the membrane in sinus lift were more frequent with the rotary technique (18\%) than with ultrasound (9\%). Implant survival and bone gain were statistically showed no significant difference between the two study groups throughout follow-up period.
\end{abstract}

\section{INTRODUCTION}

After loss of teeth in the posterior maxilla, the alveolar ridge decreases by bone atrophy and osseointegration of implants in patients with pneumatized maxillary sinuses are difficult to achieve $e^{1-7}$. Sinus elevation allows maxillary bone augmentation and thus facilitates implant rehabilitation in patients with severe posterior maxillary atrophy. In direct maxillary sinus lift

* Associate Professor, Department of Oral and Maxillofacial Surgery, Faculty of Oral and Dental Medicine, Cairo University, Egypt 
a vestibular osteotomy is performed, a bone window is prepared, and access is gained to the maxillary sinus for elevation of the membrane. The bone perforation can be carried out using an osteotomy drill in the context of the conventional rotary technique, or using ultrasound tips ${ }^{6}$.

While the sinus elevation procedure is considered by some to be a relatively invasive procedure, a comparatively low incidence of surgical and postsurgical complications of the procedure has been reported 8,9 . The most common surgical complication is the perforation of the Schneiderian membrane $2,3,6,8,10-12$. It occurs in $7-10 \%$ to $35 \%$ of sinus floor elevation procedures $1,5,7,9,10$. Membrane perforations, according to the literature, are strongly associated with the appearance of postoperative complications and consist mostly of acute or chronic sinus infection, bacterial invasion, swelling, bleeding, wound dehiscence, loss of the graft material and a disruption of normal sinus physiologic function ${ }^{2,3,5,8-13}$.

Small perforations usually do not need treatment because the membrane folds on itself during the elevation ${ }^{2-4,10}$. However, large perforations are usually managed using a bio-absorbable membrane ${ }^{2-4,7-10,12}$, by placing a large lamellar bone sheet ${ }^{4}$, using a block graft inserted of a cancellous graft $^{10}$ or by abandonment of the procedure ${ }^{1-3,9,10,14}$.

In elevation with the rotary technique, the main intraoperative complication is perforation of Schneider's membrane, which is observed in between $10-35 \%$ of all such operations ${ }^{10}$, and which usually occurs in the osteotomy drilling phase while preparing the window for access to the sinus 9 . With the purpose of reducing the risk of perforating Schneider's membrane, vestibular osteotomy using ultrasound has been proposed, as this reduces the risk of soft tissue damage ${ }^{15}$ and percentage membrane perforation to seven percent ${ }^{16}$.

Piezosurgery is based on ultrasonic principle with modulated frequency and controlled tip vibration range ${ }^{17-20}$. Selective cutting is possible with different frequencies acting only on hard tissues. It is particularly important when working in close proximity to vital anatomical structures such as nerve, vessel, or Schneiderian membrane ${ }^{15,17}$. Piezosurgery has a wide field of application in dental implantology including sinus lifting, autogenous bone harvesting, and bone crest splitting, and removing of failed implants ${ }^{18,19}$. It provides precise bone cut without much pressure, which helps to prevent excessive heat that would result in bone damage ${ }^{15,18,19}$.

CYPRESS, California--Dentium USA, a manufacturer of dental products, has introduced a sinus elevation kit called DASK (Dentium Advanced Sinus Kit). DASK Drill \#4 or \#5 as conventional rotary technique is used to prepare a lateral sinus window for sinus lifting through the antrostomy (thin-out) approach using light pressure and rotating stokes. The DASK's diamond-coated drills were designed to help prevent sinus perforation and provide an optimal irrigation function. The internal irrigation not only has a cooling effect, it also adds hydraulic pressure. This slightly lifts the sinus during the procedure. Drill speeds for DASK drills \#4 or \#5 ranges from 800 to $1,200 \mathrm{rpm}$ and 30 to $45 \mathrm{~N} . \mathrm{cm}$ with the irrigation process (http://www. dentiumusa.com).

Autogenous bone as grafting material for sinus augmentation is considered up till now as "the golden standard". However, there is a continuous search for alternative materials such as xenografts; an organic de-proteinized bovine-derived product Bio-Oss, allografts; demineralized freeze-dried bone (DFDBA) and a combination of these to form composite grafts for sinus lifting procedure 21,22 . The present study was designed to compare the intraoperative and postoperative performance of the ultrasound piezosurgery technique versus conventional DASK diamond-coated rotary technique in application to sinus lift, analyzing sinus membrane perforation in direct maxillary sinus lift with both techniques, and analyzing the bone gain obtained after the sinus augmentation based upon 
cone beam computed tomography (CBCT) scans and examining the post-augmentation survival of implants.

\section{MATERIALS AND METHODS}

\section{Patients' selection}

Twenty-two patients (16 males, 6 females) requiring dental implant therapy in the posterior maxillary region with insufficient bone volume were included in the study. Mean age was $46.10 \pm 7.20$ years. Inclusion criteria were the need for unilateral or bilateral sinus lifting with residual alveolar bone height less than $5 \mathrm{~mm}$. patients who showed any uncontrolled systemic diseases, ongoing chemotherapy or radiotherapy or a history of maxillary sinus diseases were excluded. All patients were informed of the requirements for participation in the study and signed an appropriate consent form. Only one side was included in this study, even if the patient needed bilateral sinus lifting.

The osteotomy site included one premolar and one molar tooth width in 19 patients to standardize the size of bony window opened for each study group. In three patients only the osteotomy site included one premolar and two molar teeth. A total of 22 sinus lifting surgeries were carried out and a total of 47 dental implants were placed three months after sinus augmentation to restore missing maxillary posterior tooth or teeth. Before treatment, all patients were clinically and radiographically examined by panoramic radiography and cone beam computed tomography (CBCT) scanning in selected cases for available bone volume, bone quality, anatomy and any existing sinus pathology (figure 1)

\section{Patients' grouping}

Patients were randomly allocated to Piezosurgery group (PSG) and the conventional rotative DASK group (CSG) according to the device used for preparation of the lateral sinus window. In either Piezosurgery group or conventional rotative DASK group a total of 11 sinus lifting surgeries in 11 patients were performed.

\section{Surgical technique}

All sinus lifting surgeries were performed under local anesthesia using articaine HCL 4\% with epinephrine 1: 100,000 (Septocaine, distributed by Septodont, Inc, USA). A prophylactic oral antibiotic of one gm of augmentin (875 mg amoxicillin and $125 \mathrm{mg}$ clavulanic acid, manufactured by Glaxo SmithKline, Egypt) was used, beginning 2 hours before the procedure and continued for 7 days. The sinus augmentation procedure followed the technique described by Tatum and coworkers ${ }^{14}$. A horizontal antero-posterior incision was made slightly palatal to the alveolar crest and supplemented by buccal releasing incisions at the anterior and posterior ends of the horizontal incision.

A full-thickness mucoperiosteal flap was raised and the lateral wall of the sinus was exposed. Osteotomy was performed with either Piezosurgery device (Piezosurgery, Mectron, Genova, Italy) in Piezosurgery group or rotative DASK (Dentium Advanced Sinus Kit; California-Dentium USA) drill \#4 or \#5 with drill speed 800 to $1200 \mathrm{rpm}$ with internal irrigation in the conventional rotative DASK group with copious sterile saline. Drill \#4 and \#5 with a diameter of $6.0 \mathrm{~mm}$ and $8.0 \mathrm{~mm}$ respectively and length of $2.5 \mathrm{~mm}$ has diamond coating cutting surface (figures 2,3, and 4). Sinus membrane dissection and elevation were also performed with piezosurgery tips (figure 5) or direct sinus lift elevators. Care was taken not to perforate the sinus membrane.

After obtaining sufficient space by elevating the sinus membrane, inorganic bovine bone granules (Geistlich Bio-Oss) were used for grafting. The amount of grafting material used at each site varied according to the extent of maxillary bone resorption and the sinus anatomy. Geistlich Bio-Oss is a porous bone mineral matrix. It is produced by removal of all organic components from bovine bone. It is available in spongiosa (cancellous) granules. 
The anorganic bone matrix of Geistlich Bio-Oss has macro- and microscopic structures similar to human bone. The formation and ingrowth of new bone at the implantation site of Geistlich Bio-Oss is favored, due to its trabecular architecture, interconnecting macro and micro pores and its natural consistency (https://www.geistlich-na.com).

Bony sinus windows were covered with a resorbable collagen membrane (Collagene AT, Sistema At; Italy). Mucoperiosteal flaps were primarily closed wit $3 / 0$ silk suture. Patients were advised not to blow their noses and to sneeze opening the mouth for one week after surgery. All patients were prescribed oral antibiotic of one gram augmentin twice daily for 7 days and $500 \mathrm{mg}$ paracetamol twice daily, chlorhexidine mouth rinse stating from the next day of surgery twice daily for 15 days. Sutures were removed on the seventh postoperative day.

Time from the beginning of osteotomy to the completion of sinus membrane elevation and the incidence of membrane perforation during the sinus lifting surgery were assessed to compare the effect of the two techniques. Moreover, postoperative pain and swelling were self-assesses by the patient on a 4-point scale ranging from 0 to 3 where, 0 , little/ none; 1 , some; 2 , quite a bit; and 3 very much. Patients were asked to fill out the form on $8^{\text {th }}, 24^{\text {th }}$,

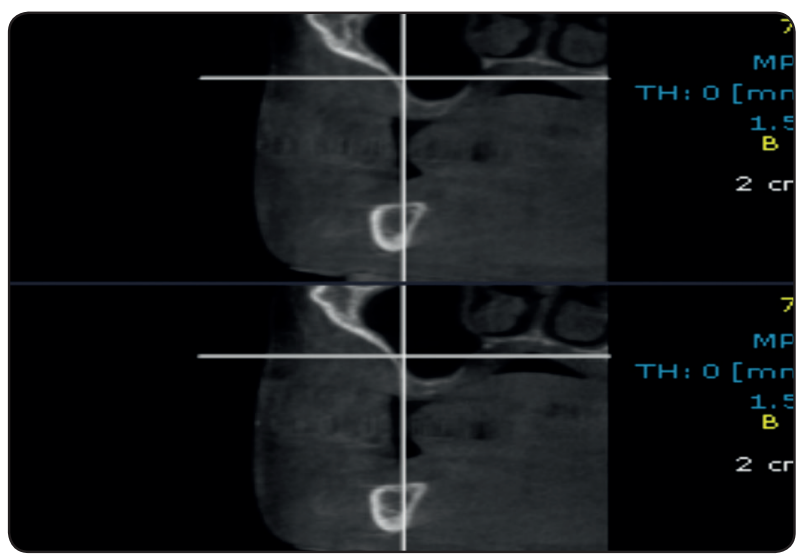

Fig. (1) Preoperative CBCT scans showing posterior maxillary resorbed alveolar ridges $48^{\text {th }}$, and $72^{\text {nd }}$ postoperative hours and on the $7^{\text {th }}$ postoperative day. CBCT scans of the surgical site immediately after sinus floor augmentation were carried out (figure 6).

\section{Delayed implant insertion}

Three months after sinus augmentation, a total of 47 Osstem TS implant system (Dental Direct UK Ltd; Osstem GmbH Hiossen Inc) of 11.5 and $13 \mathrm{~mm}$ length and $\varnothing 4.0$, and $\varnothing 5.0 \mathrm{~mm}$ diameter were inserted in target sites of maxillary premolarmolar tooth in either Piezosurgery group or rotative conventional DASK group. Then 3 months after implant insertion, implants were loaded with the final prosthesis (figures 7,8, and 9).

\section{Postoperative radiographic evaluation}

The preoperative residual bone height and the elevated bone height or bone gain after sinus floor augmentation was assessed and measured in the CBCT along the center of the proposed implant site immediately after sinus grafting as well as 3 months and 6 months after sinus floor augmentation and implant insertion. The residual bone height was measured from the top of the alveolar crest to the sinus floor. The elevated bone height was counted by subtracting between the post-surgical and initial ridge height.

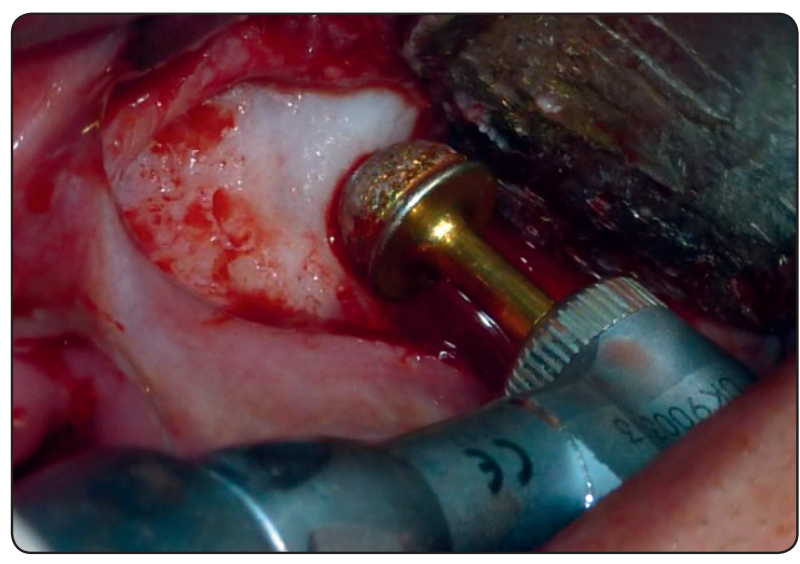

Fig. (2) DASK drill in use for creating the bony window for sinus lift 


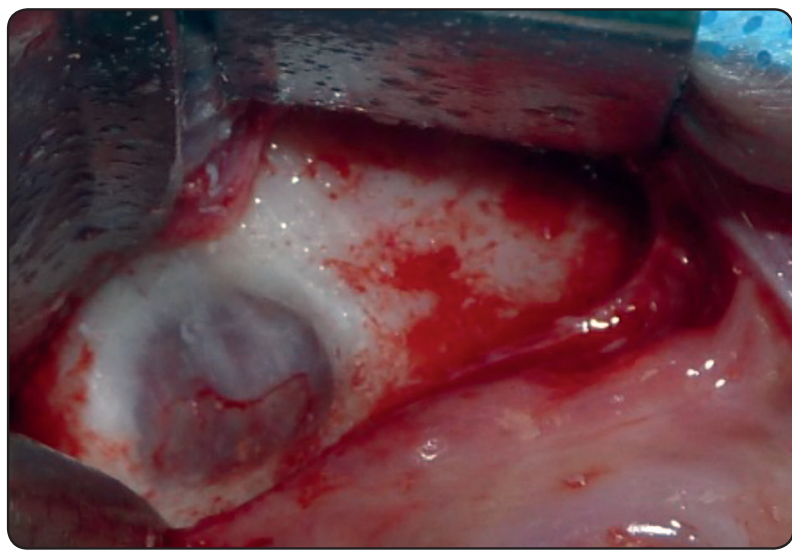

Fig. (3) Window opening using DASK drills with intact sinus membrane

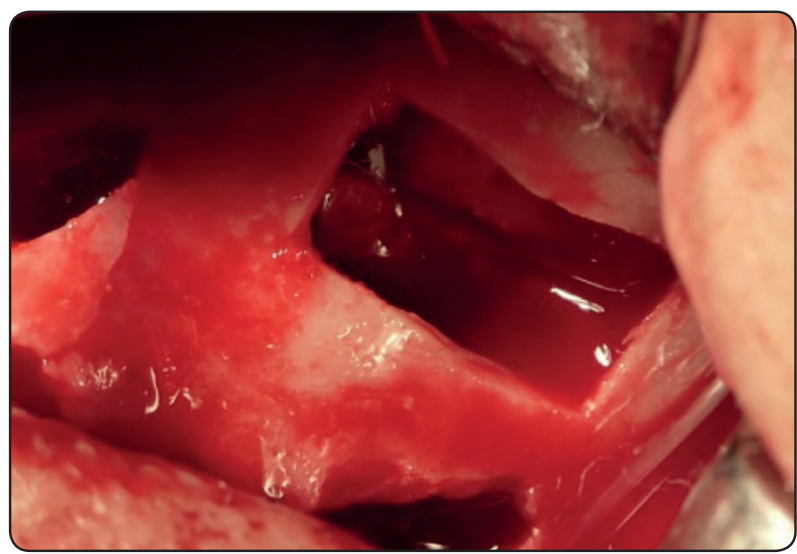

Fig. (5) Window opening using piezosurgery and lifting sinus membrane

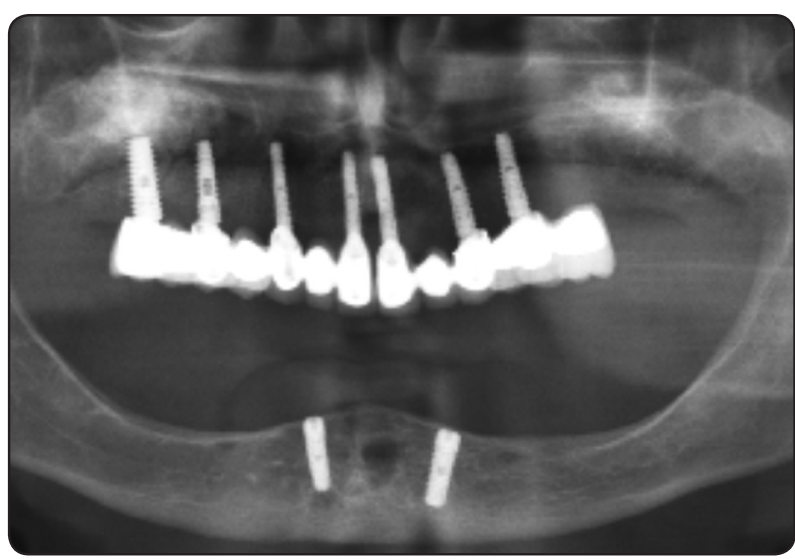

Fig. (7) Panoramic radiograph showed implants after loading

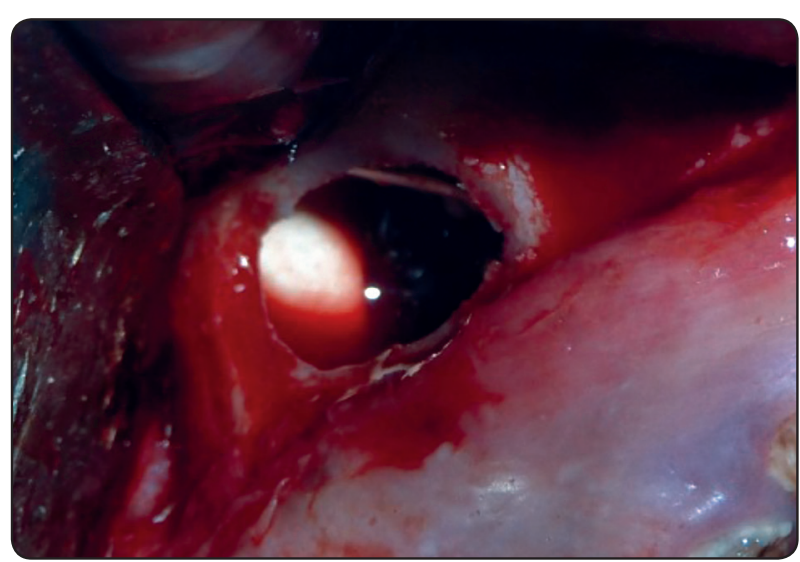

Fig. (4) Lifting sinus membrane for sinus grafting

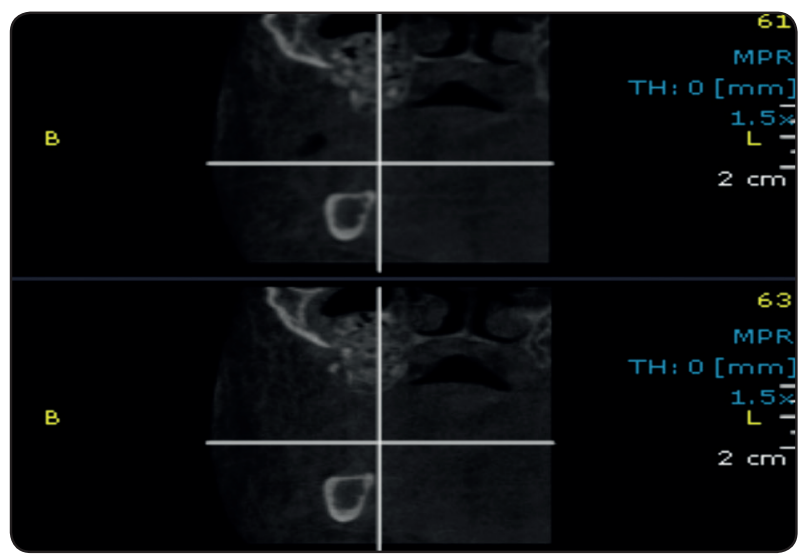

Fig. (6) CBCT scans of the surgical site immediately after sinus floor augmentation

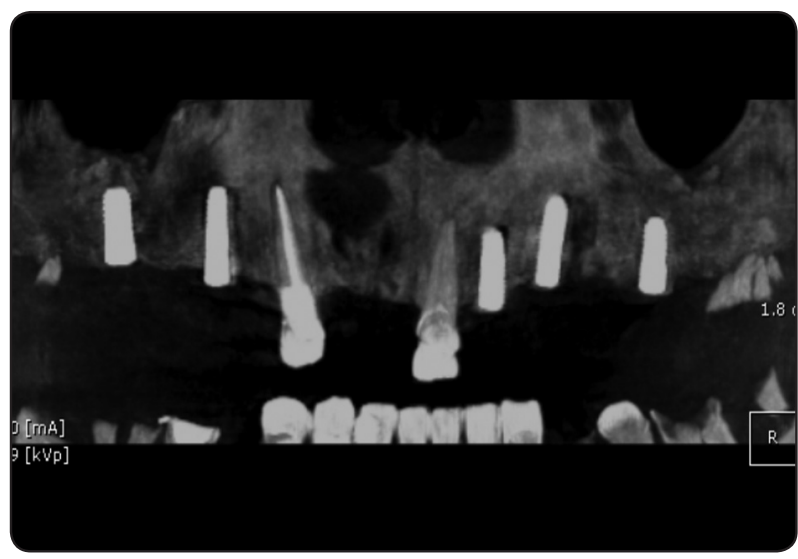

Fig. (8) CBCT scans showed implants in the bilaterally augmented maxillary sinus 


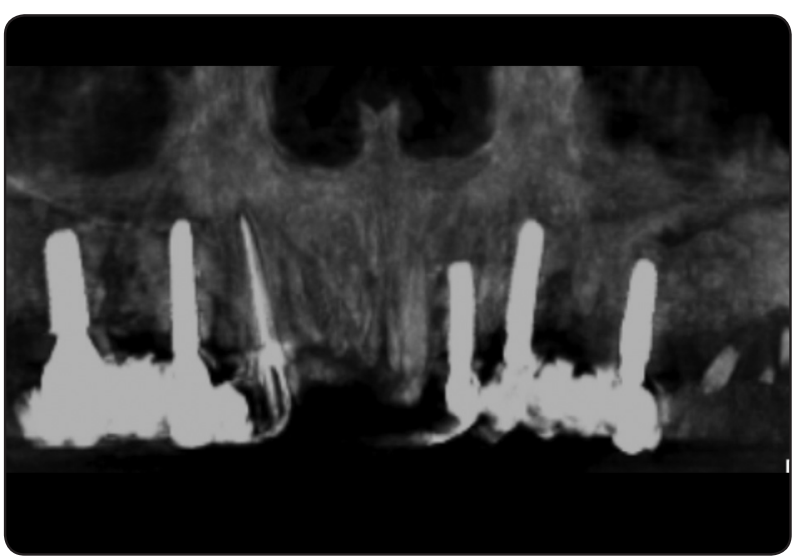

Fig. (9) CBCT scan showed constructed prosthesis on implants

\section{Statistical analysis}

Data were presented as mean and standard deviation values. Paired t-test was used to study the effect of time on each group, to compare between the two groups and to compare between the percentages changes in bone height or gain of the two study groups. Mann-Whitney test was used to compare the other parameters between the groups and Fisher exact test was used to compare incidence of membrane perforation. $\mathrm{P} \leq 0.05$ was considered significant.

\section{RESULTS}

The results of 22 patients were evaluated. The piezosurgery group consisted of 7 men and 4 women with age range of 37 to 52 years and a mean of 45.1 years. The conventional group consisted of 8 men and 3 women with age range of 36 to 53 years and a mean of 46.2 years. Only one membrane perforation occurred piezosurgery group (9\%) and two perforations occurred in conventional surgery group (18\%) during sinus lifting surgery $(9 \%$ in each group). The perforation was closed with resorbable collagen membrane and the grafting procedure was completed. There was no statistical significant difference regarding time elapsed between the beginning of osteotomy to the completion of sinus membrane elevation between piezosurgery and conventional groups $(P=0.523)$.
Pain experiences were significantly higher in the conventional group than piezosurgery group on $8^{\text {th }}$ postoperative hours $(P=0.003), 24^{\text {th }}$ postoperative hours $(P=0.018)$ and $48^{\text {th }}$ postoperative hours $(P=$ $0.017)$. However, there were no statistical significant differences between the conventional group and piezosurgery group on $72^{\text {nd }}$ postoperative hours $(P=0.129)$ and $7^{\text {th }}$ postoperative day $(P=0.412)$. There was statistical significant more postoperative swelling in the conventional group compared to piezosurgery group on the on $8^{\text {th }}$ postoperative hours $(P=0.06), 24^{\text {th }}$ postoperative hours $(P=0.02)$ and $48^{\text {th }}$ postoperative hours $(P=0.07)$. However, there were no statistical significant differences in postoperative swelling between the conventional group and piezosurgery group on $72^{\text {nd }}$ postoperative hours $(P=0.312)$ and $7^{\text {th }}$ postoperative day $(P=1.10)$. No post-operative wound dehiscence, oroantral fistula, barrier membrane exposure, and wound infection were recorded in either study groups in the current study.

Immediate CBCT scanning after sinus lifting surgeries in all patients showed proper sinus grafting. CBCT after implant insertion and 3 months after sinus grafting showed implants that inserted in the sinus cavity with dense grafted bone around them. Three months after implant insertion, CBCT showed that the sinus cavity around the implants was filled with a dense bone-like tissue. No implant was failed in both piezosurgery and conventional groups with $100 \%$ success rate.

The mean and standard deviation (SD) of the postoperative elevated bone height or bone gain in $\mathrm{mm}$ after sinus floor augmentation at different intervals in piezosurgery group and conventional group were represented in (table 1 and figure 10). There were no statistically significant differences in postoperative elevated bone height or bone gain between piezosurgery group and conventional group through all postoperative intervals. At the $6^{\text {th }}$ postoperative month after sinus grafting (3 months after implant insertion) conventional group showed a statistically significant increase in the mean bone height than piezosurgery group. The effect of time 
on the mean elevated bone height or bone gain after sinus lifting surgeries to the $6^{\text {th }}$ postoperative month in piezosurgery group and conventional group were represented in (table 2). There was significantly increase in the mean postoperative elevated bone height through all postoperative intervals in both study groups. The percentage changes in bone height were presented in (table 3and figure 11). There was no statistically significant difference between the study groups through most postoperative intervals.

TABLE (1) The means and standard deviation (SD) values and the results of paired t-test for bone height at different periods in piezosurgery group and conventional group

\begin{tabular}{|c|c|c|c|c|}
\hline Periods & $\begin{array}{c}\text { Mean } \pm \text { SD Bone height } \\
\text { in mm in CBCT Scan } \\
\text { Piezosurgery group }\end{array}$ & $\begin{array}{c}\text { Mean } \pm \text { SD Bone height } \\
\text { in mm in CBCT Scan } \\
\text { Conventional group }\end{array}$ & $t$-value & $P$-value \\
\hline Preoperative & $5.3 \pm 0.8$ & $5.1 \pm 0.7$ & 0.050 & 0.952 \\
\hline Immediately after sinus lift & $19.4 \pm 0.7$ & $19.0 \pm 1.0$ & -0.728 & 0.484 \\
\hline 3 months after sinus lift & $18.9 \pm 1.1$ & $18.6 \pm 0.8$ & -0.922 & 0.399 \\
\hline 6 months after sinus lift & $17.9 \pm 0.9$ & $17.1 \pm 0.7$ & -2.601 & $0.029 *$ \\
\hline
\end{tabular}

\section{*: Significant at $P \leq 0.05$}

TABLE (2): The means bone height in mm differences and results of paired t-test for CBCT in piezosurgery group and conventional group

\begin{tabular}{|c|c|c|c|c|}
\hline Group & Period & Mean difference & $t$-value & $P$-value \\
\hline \multirow{6}{*}{ 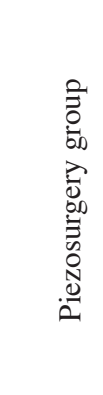 } & Preoperative - Immediate after sinus lift & -14.1 & -27.326 & $<0.001 *$ \\
\hline & Preoperative -3 months after sinus lift & -13.6 & -25.415 & $<0.001 *$ \\
\hline & Preoperative -6 months after sinus lift & -12.6 & -24.344 & $<0.001 *$ \\
\hline & Immediate after sinus lift -3 months after sinus lift & 0.5 & 4.503 & $0.001 *$ \\
\hline & Immediate after sinus lift- 6 months after sinus lift & 1.5 & 25.019 & $<0.001 *$ \\
\hline & 3 months after sinus lift -6 months after sinus lift & 1.0 & 15.323 & $<0.001 *$ \\
\hline \multirow{6}{*}{ 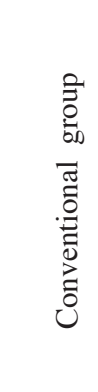 } & Preoperative - Immediate after sinus lift & -13.9 & -32.617 & $<0.001 *$ \\
\hline & Preoperative -3 months after sinus lift & -13.5 & -36.299 & $<0.001 *$ \\
\hline & Preoperative -6 months after sinus lift & -12.0 & -32.823 & $<0.001 *$ \\
\hline & Immediate after sinus lift- 3 months after sinus lift & 0.4 & 3.967 & $0.003 *$ \\
\hline & Immediate after sinus lift- 6 months after sinus lift & 1.9 & 14.000 & $<0.001 *$ \\
\hline & 3 months after sinus lift -6 months after sinus lift & 1.5 & 8.7523 & $<0.001 *$ \\
\hline
\end{tabular}


TABLE (3) The means percentage change in bone heights in CBCT in the two groups

\begin{tabular}{|l|c|c|c|c|}
\hline & $\begin{array}{c}\text { Mean \% changes in } \\
\text { piezosurgery group }\end{array}$ & $\begin{array}{c}\text { Mean\% changes in } \\
\text { conventional group }\end{array}$ & $t$-value & $P$-value \\
\hline Preoperative - Immediate after sinus lift & -266.03 & -272.5 & 0.132 & 0.881 \\
\hline Preoperative - 3months after sinus lift & -256.6 & -264.7 & 0.281 & 0.773 \\
\hline Preoperative - 6 months after sinus lift & -237.7 & -235.2 & 0.974 & 0.362 \\
\hline Immediate after sinus lift -3 months after sinus lift & 2.5 & 2.1 & 0.913 & 0.371 \\
\hline Immediate after sinus lift -6 months after sinus lift & 7.7 & 10 & 5.377 & $<0.001 *$ \\
\hline 3 months after sinus lift -6 months after sinus lift & 5.29 & 8.06 & 3.521 & $0.007 *$ \\
\hline
\end{tabular}

\section{*: Significant at $P \leq 0.05$}

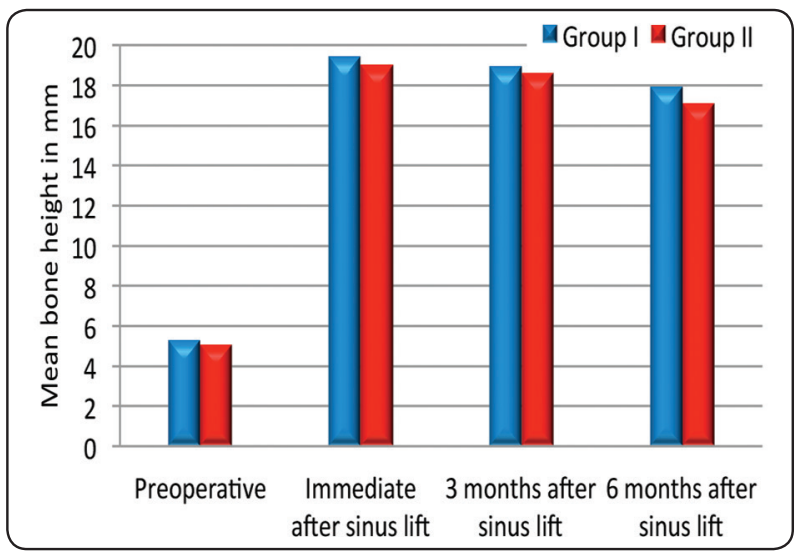

Fig. (10) Bar chart represents means bone height values in CBCT for piezosurgery group (group I) and conventional rotary group (group II)

\section{DISCUSSION}

Grafting of the maxillary sinus is a method for reaching sufficient bone height for posterior maxillary implant placement and has proven to be a highly successful method and to give predictable results $2,3,5,10,13,14$. There are different techniques and armamentaria presented to perform this surgery ${ }^{23-30}$. Oscillation frequency used in Piezosurgery is designed for acting only on mineralized tissue; therefore, the cutting tip becomes inactive when it contacts to soft tissue ${ }^{17-19,31}$. Piezosurgery is safely used in dentistry where there is high risk of damaging vital soft tissue such as nerves, vessels, and so on ${ }^{17,19,20}$.

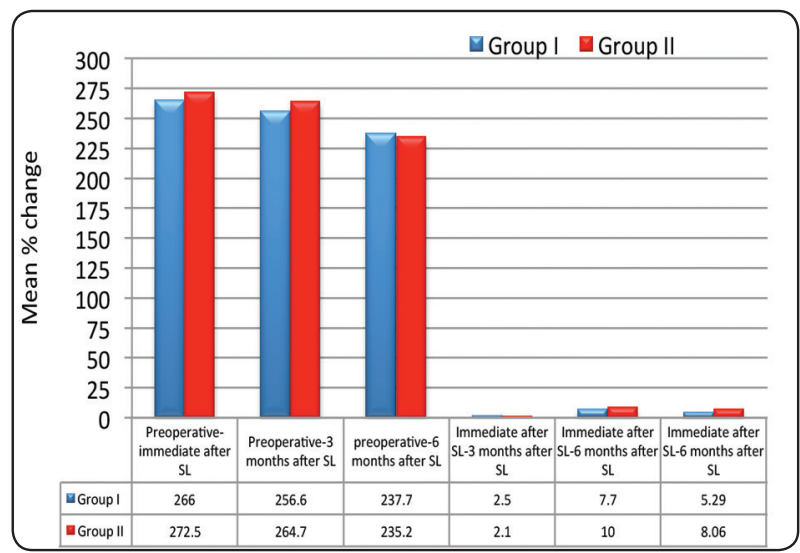

Fig. (11) Bar chart represents means percentage change in bone height values in CBCT scanning for piezosurgery group (group I) and conventional rotary group (group II)

The most commonly reported intra operative complications of sinus augmentation are membrane perforations ${ }^{4,6,8,11}$. It has been reported to occur in $7-35 \%$ of sinus floor elevation procedure 1,5 , $7,9,10$. The intact sinus membrane is essential for graft stability and prevention of sinus infection. It is reported that sinus membrane perforation risk is reduced by using Piezosurgery ${ }^{17,32}$. Wallace et al ${ }^{33}$ reported 7 membrane perforations in a total of 100 sinus lifting. All the perforations occurred while using hand instruments for membrane elevation but not during the use of Piezosurgery itself. Ultrasound is associated with fewer perforations of the membrane. In this context, Vercellotti et al ${ }^{15}$ 
performed 21 direct maxillary sinus lift procedures in 15 patients, with a perforation rate of only $5 \%$. Barone et al ${ }^{18}$ conducted a study that compared conventional drills and piezoelectric device in maxillary sinus floor elevation. They concluded that the time required for window osteotomy was higher with Piezosurgery, but membrane perforation rate was smaller compared with the conventional method ( $23 \%$ vs. $30 \%)$.

In the present study, sinus lift with the ultrasound technique afforded a higher success rate than the rotary technique. Membrane perforations were likewise less common with piezosurgery (9\% versus $18 \%$ with the conventional rotary DASK technique). In contrast, Barone et al ${ }^{34}$ treated 26 patients, 13 with the conventional rotary technique and 13 with ultrasound, and observed no statistically significant differences between the two techniques in terms of membrane perforation. Of 22 direct maxillary sinus lifts performed in the present study, a smaller number of perforations of Schneider's membrane were documented (13.6\%). In contrast, Thor et al ${ }^{35}$ in 20 patients performed 27 direct maxillary sinus lift procedures with the rotary technique. There were 11 membrane perforations ( $41 \%$ of the overall operations). Using the same technique, Swartz-Arad et al ${ }^{9}$ obtained a similar result; with 36 perforations in 81 maxillary sinuses lift procedures ( $44 \%$ of the operations). Ultrasound is associated with fewer perforations of the membrane.

Repairing of the perforations can be challenging due to size of the perforation, and there is a risk of surgical failure ${ }^{12,36,37}$. In all cases of perforation of the sinus membrane we placed a reabsorbable collagen membrane, as indicated by HernandezAlfaro et al ${ }^{38}$ for perforations measuring less than $5 \mathrm{~mm}$ in size. Some authors considered that tearing the Schneiderian membrane was a factor that diminished the implant survival rate: Proussaefs et al ${ }^{12}$ obtained a lower implant survival rate when they were placed in the sinus lifts with perforated membrane $(69.5 \%)$, than when they were placed in sinus lifts with intact membrane $(100 \%)$. Khoury ${ }^{1}$ also found a lower survival rate for implants in the interventions in which perforation of the sinus membrane took place. Some authors 39, 40 did not consider the tearing of the membrane a negative factor in the survival rate of the implant. Schwartz-Arad et al ${ }^{9}$ considered that the tearing of the membrane influenced the occurrence of post-surgical complications, but did not influence the survival rate of the implants. Interestingly, in this study, all the 47 implants had been integrated properly with no complications.

There is a general agreement in the literature regarding the longer time period required for operations with the Piezosurgery device ${ }^{17,19,32}$. However, in our study, although the time for osteotomy and membrane elevation was longer in the Piezosurgery group than the conventional group, the difference was not statistically significant. Piezosurgery produces less vibration and noise as it uses micro vibrations, in contrast to macro vibrations and the noise produced by conventional rotary technique ${ }^{15,17}$. This makes the Piezo-system more manageable and allows greater intraoperative control. Heinemann et al ${ }^{17}$ and Torrella et al ${ }^{24}$ stated that Piezosurgery provides more comfort to the patient and to the practitioner during the operation and causes less morbidity and complications compared with conventional rotary methods. It is claimed that clear operation site can be provided by using the Piezosurgery device ${ }^{18,20}$. It maintains a blood-free surgical field during bone cutting due to air-water cavitation effect of the ultrasonic device ${ }^{15,26}$.

In this study, postoperative pain and swelling were significantly less in Piezosurgery group than the conventional group. Pain and swelling are the most encountered complications due to the nature of bone surgery, and intraoperative trauma to bone tissue is the most prominent causative factor. In the current study, the ultrasonic nature as well as more precise cut and less pressure during bone manipulation with the Piezosurgery hand piece provided less pain and swelling postoperatively. 
Regarding bone gain, Vercellotti et al ${ }^{15}$ compared the bone regeneration achieved with the ultrasound technique versus the rotary technique. To this effect the authors performed a series of ostectomies with the two techniques, in one same dog. After 56 days of follow-up, greater bone regeneration was noted in the operations performed with ultrasound. In our study, the bone gain achieved after the operation and 3 months after the operation at the time of implant insertion as well as 6 months after the operation at the time of implant loading showed statistically no significant difference when comparing piezosurgery group with conventional group.

\section{CONCLUSION}

Perforations of the sinus membrane are more frequent in direct sinus lift when using the conventional rotary technique (18\%) than with ultrasound (9\%), with no significant difference in both survival of the implants and the bone gain in both study groups. Sinus lifting procedure performed with Piezosurgery causes less pain and swelling postoperatively compared with the conventional rotary technique. Ultrasound technique allows for precise removal of bone with minimal risk of injury or perforation of the Schneider membrane. The DASK diamond-coated rotary technique reduces the incidence of sinus membrane perforation and provides an optimal irrigation function. CBCT assesses accurately the bone gain following sinus floor augmentation procedure.

\section{REFERENCES}

1. Khoury, F. (1999) Augmentation of the sinus floor with mandibular bone blocks and simultaneous implantation: a 6-year clinical investigation. International Journal of Oral \& Maxillofacial Implants 14: 557-564.

2. Bergh van den, J.P.A., ten Bruggenkate, C.M., Disch, F.J.M. \& Tuinzing, D.B. (2000a) Anatomical aspects of sinus floor elevations. Clinical Oral Implants Research 11: 256-265.

3. Bergh van den, J.P.A., ten Bruggenkate, C.M., Groeneveld, H.H.J., Burger, E.H. \& Tuinzing, D.B. (2000b)
Recombinant human bone morphogenetic protein-7 in maxillary sinus floor elevation surgery in 3 patients compared to autogenous bone grafts. A clinical pilot study. Journal of Clinical Periodontology 27: 627-636.

4. Vlassis, J.M. \& Fugazzotto, P.A. (1999) A classification system for sinus membrane perforations during augmentation procedures with options for repair. Journal of Periodontology 70: 692-699.

5. Nkenke, E., Schlegel, A., Schultze-Mosgau, S., Neukam, F.W. \& Wiltfang, J. (2002) The endoscopically controlled osteotome sinus floor elevation: a preliminary prospective study. International Journal of Oral\&Maxillofacial Implants 17: 557-566.

6. Sornı’, M., Guarino’’ s, J., Garcı’a, O. \& Pen ${ }^{\sim}$ arrocha, M. (2005) Implant rehabilitation of the atrophic upper jaw: a review of the literature since 1999. Medicina Oral, Patologı́a Oral y Cirugı'a Bucal 10: E45-E56.

7. Stricker, A., Voss, P.J., Gutwald, R., Schramm, A. \& Schmelzeisen, R. (2003) Maxillary sinus floor augmentation with autogenous bone grafts to enable placement of SLAsurfaced implants: preliminary results after 15-40 months. Clinical Oral Implants Research 14: 207-212.

8. Cho, S.C., Wallace, S.S., Froum, S.J. \& Tarnow, D.P. (2001) Influence of anatomy on Schneiderian membrane perforations during sinus elevation surgery: three-dimensional analysis. Practical Procedures \& Aesthetic Dentistry 13: $160-163$.

9. Schwartz-Arad, D., Herzberg, R. \& Dolev, E. (2004) The prevalence of surgical complications of the sinus graft procedure and their impact on implant survival. Journal of Periodontology 75: 511-516.

10. Shlomi, B., Horowitz, I., Kahn, A., Dobriyan, A. \& Chaushu, G. (2004) The effect of sinus membrane perforation and repair with Lambone on the outcome of maxillary sinus floor augmentation: a radiographic assessment. International Journal of Oral \& Maxillofacial Implants 19: $559-562$.

11. Levin, L., Herzberg, R., Dolev, E. \& Schwartz-Arad, D. (2004) Smoking and complications of onlay bone grafts and sinus lift operations. International Journal of Oral \& Maxillofacial Implants 19: 369-373.

12. Proussaefs P, Lozada J, Kim J, et al. Repair of the perforated sinus membrane with a resorbable collagen membrane: A human study. Int J Oral Maxillofac Implants. 2004; 19:413-420. 
13. Aimetti,M., Romagnoli, R., Ricci, G. \& Massei, G. (2001) Maxillary sinus elevation: the effect of macrolacerations and microlacerations of the sinus membrane as determined by endoscopy. International Journal of Periodontics and Restorative Dentistry 21: 581-589.

14. Chanavaz, M. (1990) Maxillary sinus: anatomy, physiology, surgery, and bone grafting related to implantology eleven years of surgical experience (1979-1990). Journal of Oral Implantology 16: 199-209.

15. Vercellotti T, De Paoli S, Nevins M. The piezoelectric bony window osteotomy and sinus membrane elevation: Introduction of a new technique for simplification of the sinus augmentation procedure. Int J Periodontics Restorative Dent. 2001;21: 561-567.

16. Vercellotti T, Nevins ML, Kim DM, Nevins M, Wada K, Schenk RK, et al. Osseous response following respective therapy with piezosurgery. Int J Periodontics Restorative Dent. 2005; 25:543-9.

17. Heinemann F, Hasan I, Kunert-Keil C, et al. Experimental and histological investigations of the bone using two different oscillating osteotomy techniques compared with conventional rotary osteotomy. Ann Anat. 2012; 194:165-170

18. Barone A, Santini S, Marconcini S, et al. Osteotomy and membrane elevation during the maxillary sinus augmentation procedure. A comparative study: Piezoelectric device vs. conventional rotative instruments. Clin Oral Implants Res. 2008; 19:511-515.

19. Pavlíková G, Foltán R, Horká M, et al. Piezosurgery in oral and maxillofacial surgery. Int J Oral Maxillofac Surg. 2011; 40:451-457.

20. Seshan H, Konuganti K, Zope S. Piezosurgery in periodontology and oral implantology. J Indian Soc Periodontol. 2009;13:155-156.

21. Cagri D, Gokhan G,: Comparison of piezosurgery and conventional rotative instruments in direct sinus lifting. Implant Dent 2013; 22:662-665

22. María P, Cristina S, David P, Javier R: Osteotomy in direct sinus lift. A comparative study of the rotary technique and ultrasound. Med Oral Patol Oral Cir Bucal. 2012 1; 17 (3): e457-61.

23. Baldi D, Menini M, Pera F, et al. Sinus floor elevation using osteotomes or piezoelectric surgery. Int J Oral Maxillofac Surg. 2011;40:497-503.
24. Torrella F, Pitarch J, Cabanes G, et al. Ultrasonic ostectomy for the surgical approach of the maxillary sinus: A technical note. Int J Oral Maxillofac Implants. 1998;13:697-700.

25. Bruschi GB, Crespi R, Capparè $P$, et al. Transcrestal sinus floor elevation: A retrospective study of 46 patients up to 16 years. Clin Implant Dent Relat Res. 2012; 14:759-767.

26. Schlee M, Steigmann M, Bratu E, et al. Piezosurgery: Basics and possibilities. Implant Dent. 2006;15:334-340.

27. Esposito M, Grusovin MG, Rees J, et al. Effectiveness of sinus lift procedures for dental implant rehabilitation: A Cochrane systematic review. Eur J Oral Implantol. 2010;3:7-26.

28. Cassetta M, Ricci L, Iezzi G, et al. Use of piezosurgery during maxillary sinus elevation: Clinical results of 40 consecutive cases. Int J Periodontics Restorative Dent. 2012;32:e182-e188.

29. Sohn DS, Moon JW, Lee WH, et al. Comparison of new bone formation in the maxillary sinus with and without bone grafts: Immunochemical rabbit study. Int J Oral Maxillofac Implants. 2011;26: 1033-1042.

30. Trombelli L, Franceschetti G, Rizzi A, et al. Minimally invasive transcrestal sinus floor elevation with graft biomaterials. A randomized clinical trial. Clin Oral Implants Res. 2012;23:424-432.

31. Pippi R, Alvaro R. Piezosurgery for the lingual split technique in mandibular third molar removal: A suggestion. $\mathrm{J}$ Craniofac Surg. 2013;24:531-533.

32. Happe A. Use of a piezoelectric surgical device to harvest bone grafts from the mandibular ramus: Report of 40 cases. Int J Periodontics Restorative Dent. 2007; 27:241-249.

33. Wallace SS, Mazor Z, Froum SJ, et al. Schneiderian membrane perforation rate during sinus elevation using piezosurgery: Clinical results of 100 consecutive cases. Int J Periodontics Restorative Dent. 2007;27:413-419.

34. Barone A, Santini S, Sbordone L, Crespi R, Covani U. A clinical study of the outcomes and complications associated with maxillary sinus augmentation. Int J Oral Maxillofac Implants. 2006;21:81-5.

35. Thor A, Sennerby L, Hirsch JM, Rasmusson L. Bone formation at the maxillary sinus floor following simultaneous elevation of the mucosal lining and implant installation without graft material: an evaluation of 20 patients treated with 44 Astra Tech implants. J Oral Maxillofac Surg. 2007;65:64-72 
36. Testori T, Wallace SS, Del Fabbro M, et al. Repair of large sinus membrane perforations using stabilized collagen barrier membranes: Surgical techniques with histologic and radiographic evidence of success. Int J Periodontics Restorative Dent. 2008;28:9-17.

37. Pikos MA. Maxillary sinus membrane repair: Update on technique for large and complete perforations. Implant Dent. 2008;17:24-31.

38. Hernández-Alfaro F, Torradeflot MM, Marti C. Prevalence and management of Schneiderian membrane perfora- tions during sinuslift procedures. Clin Oral Implants Res. 2008;19:91-8.

39. Ardekian L, Oved-Peleg E, Mactei EE, Peled M. The clinical significance of sinus membrane perforation during augmentation of the maxillary sinus. J Oral Maxillofac Surg. 2006;64:277-82.

40. Karabuda C, Arisan V, Hakan O. Effects of sinus membrane perforations on the success of dental implants placed in the augmented sinus. J Periodontol. 2006; 77:1991-7. 